\title{
HIL Simulation of Aircraft Thrust Reverser Hydraulic System in Modelica
}

\author{
Zhao Jianjun1 Li Ziqiang1 Ding Jianwan1 Chen Liping1 Wang Qifu1 \\ Lu Qing2 WangHongxin2 Wu Shuang2 \\ 1: CAD Centre, Mechanical School, Huazhong Univ. Sci.\& Tech. Wuhan, Hubei, China, 430074 \\ 2: Shanghai Aircraft Design and Research Institute, Commercial Aircraft Corp. of China Ltd., \\ Shanghai, 200436 \\ \{jjzhao168,willhave, jwdingwh, chenliping.ty\}@gmail.com wangqf@hust.edu.cn \\ 1q70300@126.comwhongxin@sina.com wushuanga@sohu.com
}

\begin{abstract}
This article describes a solution to create a hardwarein-the-loop (HIL) simulation system of civil aircraft thrust reverser with Modelica-based simulation platform -- MWorks in Windows system. The HIL system uses simulation platform "MWorks" to model and simulate the thrust reverser hydraulic system, and takes hardware -- PLC's output signals as the inputs of the simulation. Modeling module, communication module, solving module, animation module and HIL control module are included in the simulation platform, whose key technology and implementation details are specified. The HIL system has been successfully applied to the simulation of ARJ21 aircraft thrust reverser hydraulic system. It can simulate the hydraulic system in normal status, fault status as well as other working conditions to verify control logic and evaluate key performance of the system, thereby helping to reduce the cost of experiments and to optimize the design of the system.
\end{abstract}

Keywords: Aircraft thrust reverser hydraulic system, real-time simulation, HIL, Modelica

\section{Introduction}

Thrust reverser [1] as a part of aircraft engine, is aircraft landing deceleration device, which can effectively shorten the distance of taxiing. Thrust reverser is a typical complex physical system, involving mechanical, electronic, hydraulic, control and other domains. In order to verify thrust reverser's control logic, we could carry out ground experiment and flight experiment with real pieces of the thrust reverser, but this approach has high cost and poor security, and it is limited to different natural conditions. Moreover, with this approach, the test for extreme condition is very difficult.
Modelica-based HIL simulation system can resolve above-mentioned problems. Firstly, Modelica [2, 3] is a freely available, object-oriented language for modeling of large, complex, and heterogeneous physical systems. It is suited for multi-domain modeling. Models in Modelica are mathematically described by differential, algebraic and discrete equations. In Modelica we can model the entire thrust reverser, which involves mechanical, electronic, hydraulic and control domains. Secondly, HIL system uses both real logic control components and thrust reverser model to implement the simulation. This HIL system can verify the control logic in a variety of working conditions, and its cost is very low. Moreover, with this system, there is no need to consider the security.

This article introduces a solution to create an HIL simulation system of thrust reverser with Modelicabased simulation platform - MWorks [4] in common computer with Windows operating system. It use as an example the aircraft thrust reverser of Advanced Regional Jet for the 21st Century (ARJ21) which is designed and manufactured by Commercial Aircraft Corp. of China, Ltd. (COMAC). At first, it introduces the overall frame of the HIL simulation system, and then specifies several key modules of the simulation platform, which are modules of modeling, solving, communication, animation and HIL control, and finally demonstrates a successful application of this system in ARJ21 thrust reverser simulation.

\section{System Overview}

Generally, HIL simulation system is composed of host PC running on Windows operating system and target machine running on real-time operating sys- 
tem. This kind of system has high real-time capability, but is very expensive.

ARJ21 aircraft thrust reverser is driven by a hydraulic system, which is mainly controlled by six electromagnetic hydraulic valves, whose states all depend on the thrust reverser control switch. In the simulation, PLC as the thrust reverser controller generates 6 hydraulic valve control signals according to the state of the thrust reverser control switch and feedback signal from simulation platform. And the feedback signal will be only used for fault trigger. Therefore, the simulation does not need very high real-time capability.

The HIL simulation system, discussed in this article, does not need expensive "true" real-time system. It can run on general computer with Windows operating system and the sampling frequency can achieve $50 \mathrm{~Hz}$, which is enough for the requirements of the thrust reverser simulation.

In Figure 1 the system overview is shown. The HIL simulation system is implemented based on PLC and simulation platform "MWorks", which consists of five software modules -- modeling module, solving module, communication module, animation module and HIL control module.

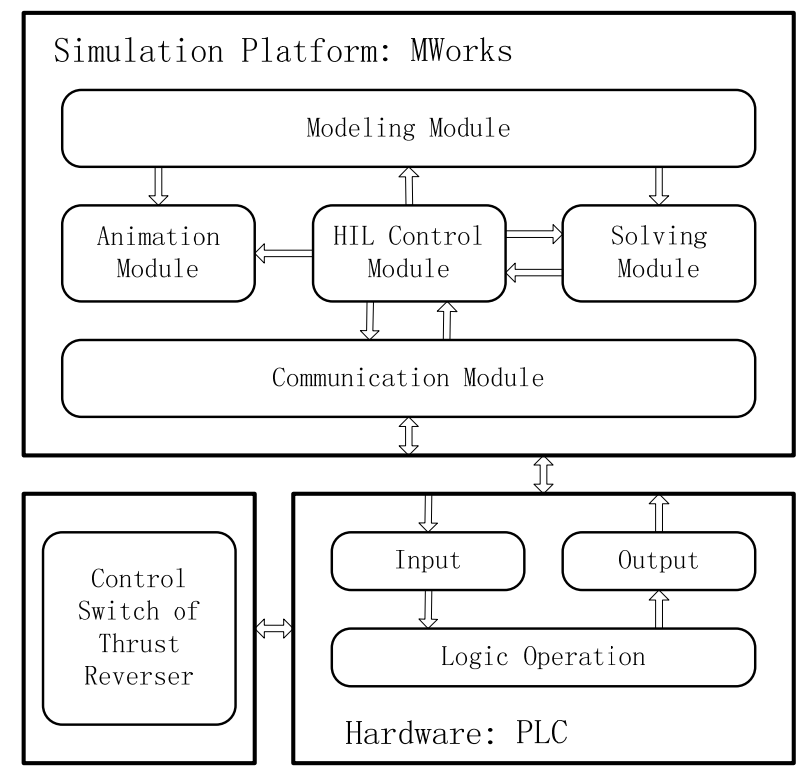

Figure 1: System overview

The PLC, used as the hardware part in the HIL system, receives electrical signal of control switch as well as simulation feedback signal, and sends control signal to the simulation platform after logic operation.
MWorks, a Modelica-based integrated development environment, is used as modeling and simulation platform for the HIL simulation system. The thrust reverser is the simulated object, which is modeled in Modelica. According to the model, the solving module generates the solver, which is responsible for real-time calculation. The communication module is responsible for real-time data exchange between simulation platform and the PLC. The animation module receives the result data from the solving module and drives 3D animation. The HIL control module, whose panel is shown in Figure 2, is responsible for starting and terminating the simulation, setting simulation parameters, displaying key data as well as communicating with other modules.

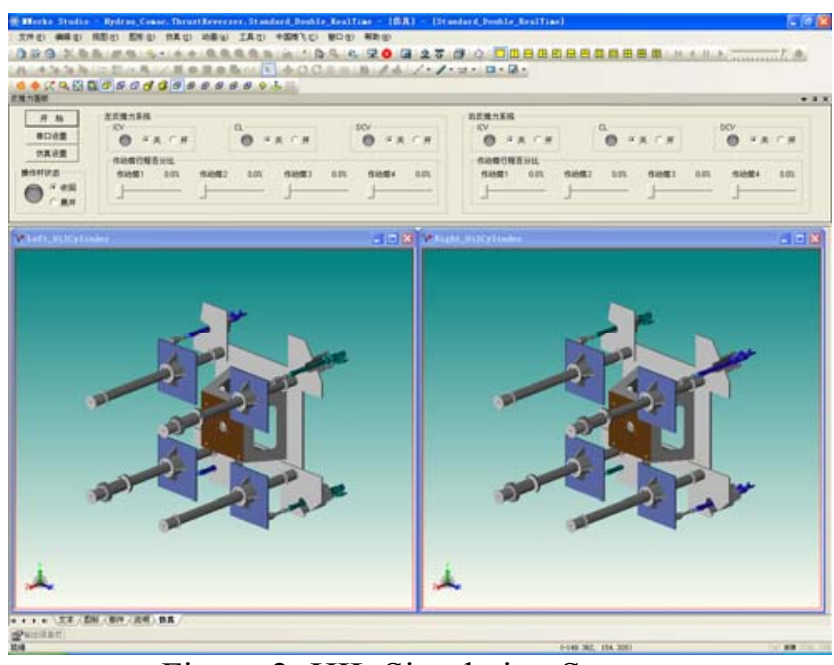

Figure 2: HIL Simulation System

The simulation process is as follows:

1) After analyzing the thrust reverser system, component models and system models are created in Modelica.

2) After setting simulation parameters with the panel of the HIL control module, the simulation begins: the HIL control module translates the model, and then the solving module generates a solver, which will be called in a new process.

3) The communication module is called by the HIL control module to receive control signals from PLC. After translating, these signals will be displayed on the panel, and sent to the solver process.

4) The solver process receives control signal and calculates in every cycle. When the calculation finishes, the solver sends the results to the HIL control module, and wait until the next cycle. 
5) The HIL control module receives the results from the solver process and displays them on the panel of the HIL control module, and delivers them to the animation module to drive real-time animation. At the same time, the HIL control module calls the communication module to send the results as feedback signal to PLC.

6) PLC uses the feedback signals and the state of control switch as input, and after logic operation, sends the control signal to the simulation platform.

7) Repeat the cycle from Step 3 until the termination of the simulation.

\section{Key Technologies}

\subsection{Modeling}

After analyzing ARJ21 aircraft thrust reverser hydraulic systems, we developed an exclusive hydraulic library: Hydrau Comac, which is based on HyLibLight hydraulic library. Hydrau_Comac library provides ARJ21 thrust reverser hydraulic components and auxiliary library, such as Isolation Control Valve (ICV), Cowl Lock (CL), Directional Control Valve (DCV), hydraulic actuator, pipe, loads, and characteristics of fluid. These models are constructed according to their physical equations with their parameters calibrated by test results if necessary. To satisfy the requirements of the real-time capability, Hydrau_Comac library also provides simplified realtime component models. The structure of Hydrau_Comac library is shown in Figure 3.

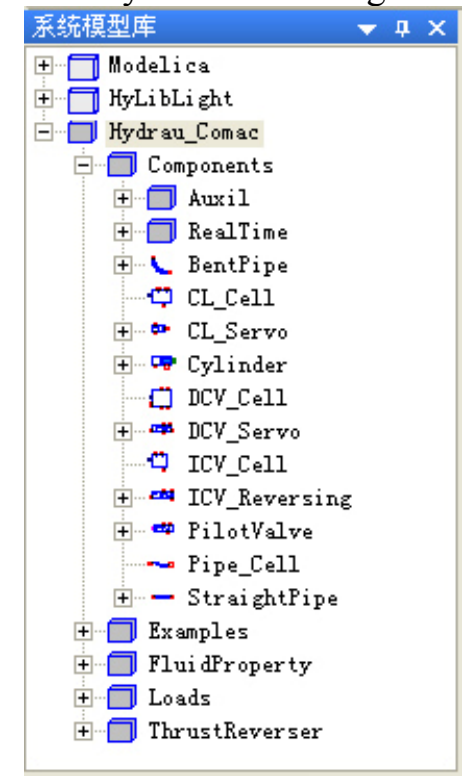

Figure 3: Structure of Hydrau_Comac library
Based on HyLibLight library and Hydrau_Comac library, we modeled ARJ21 thrust reverser hydraulic system, provided simplified system model (Figure 4) for real-time HIL simulation, as well as detailed system model (Figure 5) for off-line simulation.

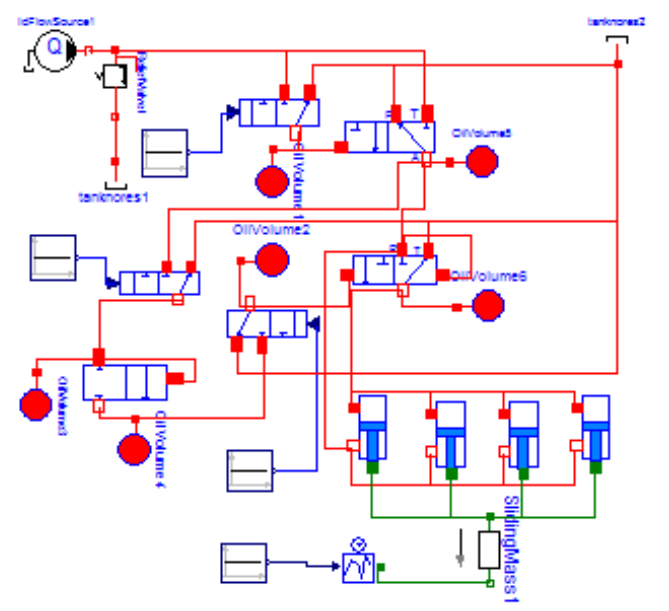

Figure 4: Real-Time System Model for Thrust Reverser

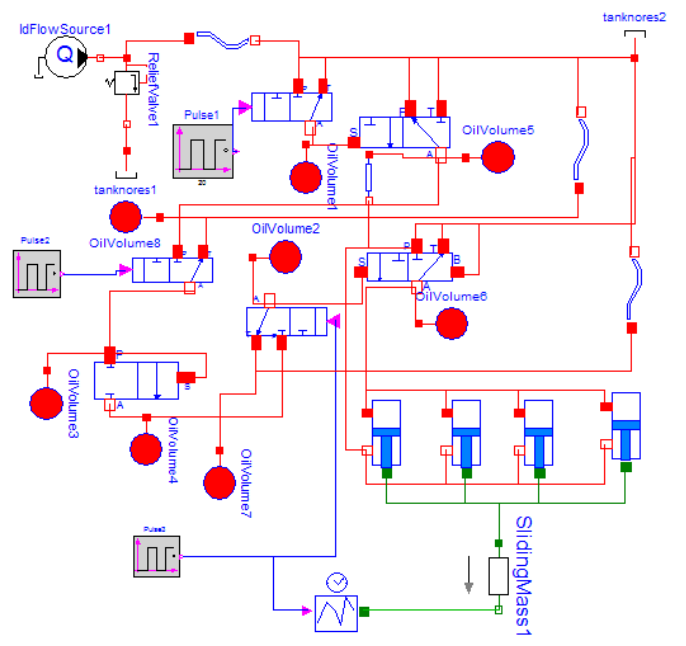

Figure 5: Off-line System Model with Pipes

\subsection{Solving}

Model solving in HIL simulation is different from in off-line simulation. The solving in HIL simulation needs to not only exchange data with external hardware, but also guarantee the synchronicity between physical time in real world and logic time in simulation.

In order to identify input and output data, we used "input" prefix and "output" prefix to modify input variables and output variables, thus we can ensure the order of the calculation -- from the input variables to output variables. Besides, according to Modelica specification, input variables and output variables are not only used for external communica- 
tion, therefore external exchange data needs to be recorded in configuration file.

According to the records in configuration file, the solving module associates input/output variables with shared memory. The solver module reads input data from shared memory, and writes output data into there. The HIL control module writes input data coming from PLC into sharing memory, and reads output data from there.

The flow chart of real-time solving is shown in figure 6 . In every sampling cycle, the solving module gets the input variables from sharing memory, and checks if their value changes, if changes, it means that there is changes in the outside world, which results in an event, so that the solving module need to do event iteration. Then the solving module calculates, and writes required output data into shared memory.

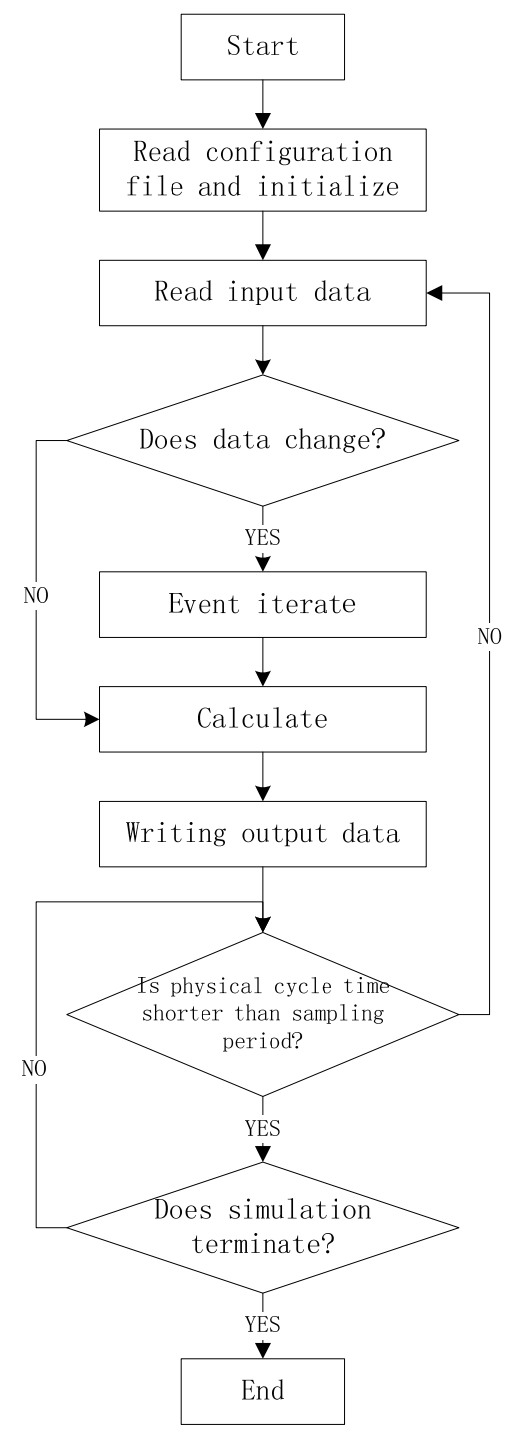

Figure 6: Flow Chart of Real-time Solving
We use timer to implement the synchronicity. By calling QueryPerformanceFrequency() function, we can obtain machine internal timer's clock frequency, and by calling QueryPerformanceCounter() function at two time points, we can get a count. With the frequency and the count, we can know the precise time between that two time points. With this method, we can know the time spent in one cycle, and the time is called physical cycle time, which is a variable. The next cycle begins when the physical cycle time is longer than sampling period. The timing error of this method is less than $1 \mathrm{~ms}$.

In every cycle, the solving module checks whether the time spent on calculating is longer than the sampling period. If the calculation overruns the sampling period, but not more than the acceptable time, the module will report a warning. And if the calculation overruns the acceptable time, the module will report an error and quit. Therefore, in order to achieve high real-time capability, the simulation system needs to run on high-performance computer to ensure the speed of solving.

\subsection{Communication}

In HIL simulation, how to communicate between simulation platform and PLC and how to guarantee the precise communication frequency are key factor to real-time capability.

By using the communication module, simulation platform communicates with PLC through RS232 serial port . Communication parameters are as follows: $57.6 \mathrm{kbps}$ transmission rate, 8-bit data bit, 1-bit stop bit, no parity, and fixed word length data frame. The data transmitted from simulation platform to PLC will be converted to standard data frame according to the protocol. After receiving, the PLC will translate those data frames to retrieve the content.

The communication module calls Windows API function to carry out serial port communication: calling CreateFile() function to open the serial port, WriteFile() function to write data to the serial port, ReadFile() function to read data from serial port.

PLC uses high-speed serial port communication module CP341 to implement communication. FB7 function block of CP341 are responsible for receiving data from simulation platform, and FB8 function block of CP341 are responsible for sending data to simulation platform. 
By using timer, the frequency of serial port communication can be controlled. Serial port communication frequency is the same as the sampling frequency. PLC uses its internal timer, whose minimum timing interval can be $10 \mathrm{~ms}$. Since the PLC is circuit working, so the precision of timing depends on the operational cycle of PLC control program. Under normal circumstances, the operational cycle of PLC control program can be less than $1 \mathrm{~ms}$, and the precision can achieve $1 \mathrm{~ms}$. The communication module, based on Windows operating system, uses multimedia timer "timeSetEvent()" for timing control, and implements serial port reading and writing operation in callback function, the precision can also achieve $1 \mathrm{~ms}$.

\subsection{Animation}

Generally, the implementation of Modelica multibody animation has 3 steps: firstly, the solver calculates the model to generate result data, which then will be used to form animation data; secondly, geometric models are created; thirdly, the geometric models are driven by the animation data and displayed on the screen.

For the real-time simulation, we need to fresh the animation data in every cycle, but it takes so long to fresh the data that the animation cannot satisfy realtime requirements. Fortunately, the thrust reverser has only one motion freedom, that is, the actuation can move back and forth. Therefore, we can create off-line animation at first, and then use the variable of actuator deployed length to control the display of that off-line animation, thus the synchronicity of the animation can be guaranteed.

Specific process is as follows: Firstly, establish the multi-body kinematic model of the thrust reverser, and execute off-line simulation to generate simulation results document; secondly, read the simulation results document to create $3 \mathrm{D}$ animation; thirdly, establish one to one mapping relationship between the variable of actuator deployed length and the offline animation frames; finally, carry out the real-time simulation, obtain the value of that variable, and use it to drive the animation.

\section{Application}

This HIL simulation system has been successfully applied to the simulation of ARJ21 aircraft thrust reverser hydraulic system. The simulation platform $\mathrm{UI}$ is shown in Figure 7.
Logic control hardware part is implemented with Siemens S7-300 series PLC, which includes power supply module, CPU module, discrete input module, discrete output module, analog input module, analog output module, serial port communication module and touch panel. PLC control program is developed with STEP7, and touch screen interface (Figure 7) is developed with Flexcible2005. PLC takes the thrust reverser control switch or the data from the touch screen as input signal, after some logic operation, it sends the output data as control signal to simulation platform.

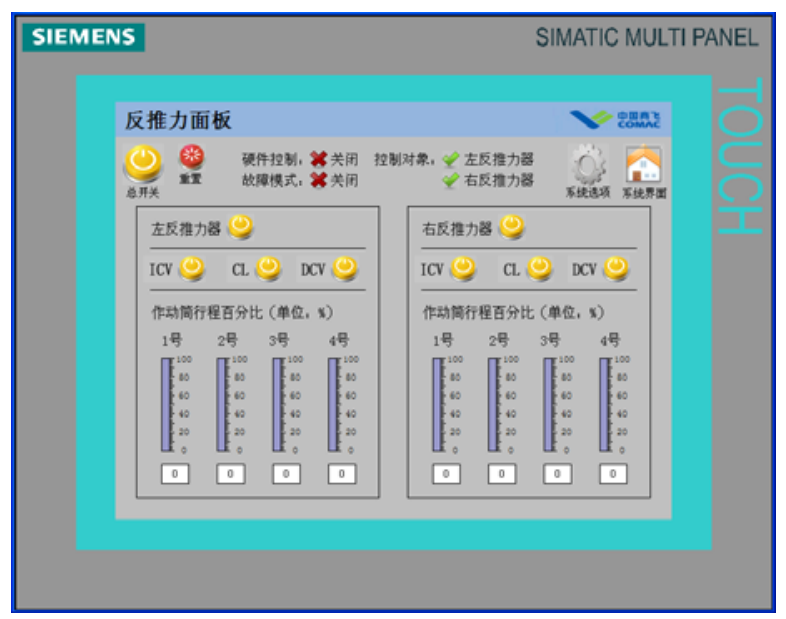

Figure 7: Touch Panel of PLC

MWorks runs on general computer with Windows operating system. our computer with simulation platform MWorks is a Dell desktop with Intel Core2 2.8G CPU, 2G RAM, ATI 3450HD graphics card and 19-inch liquid crystal display. In this configuration, the real-time simulation cycle of ARJ21 thrust reverser hydraulic system can achieve $20 \mathrm{~ms}$.

The result data and curves generated by this HIL simulation system are basically in agreement with the tests, the difference is acceptable. (Table 1, Figure 8, Figure 9).

Table 1: Deploying Time and Stowing Time of Actuator

\begin{tabular}{|c|c|c|}
\hline & $\begin{array}{c}\text { Deploying } \\
\text { Time (s) }\end{array}$ & $\begin{array}{c}\text { Stowing } \\
\text { Time(s) }\end{array}$ \\
\hline Experiment & 1.08 & 2.68 \\
\hline Simulation & 1.04 & 2.66 \\
\hline Error & $3.7 \%$ & $0.7 \%$ \\
\hline
\end{tabular}




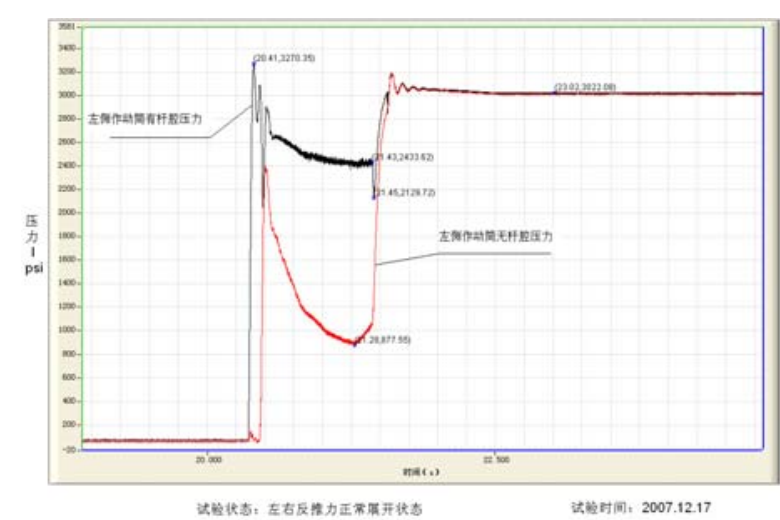

Figure 8: Experimental Curves of Pressure of The Actuator

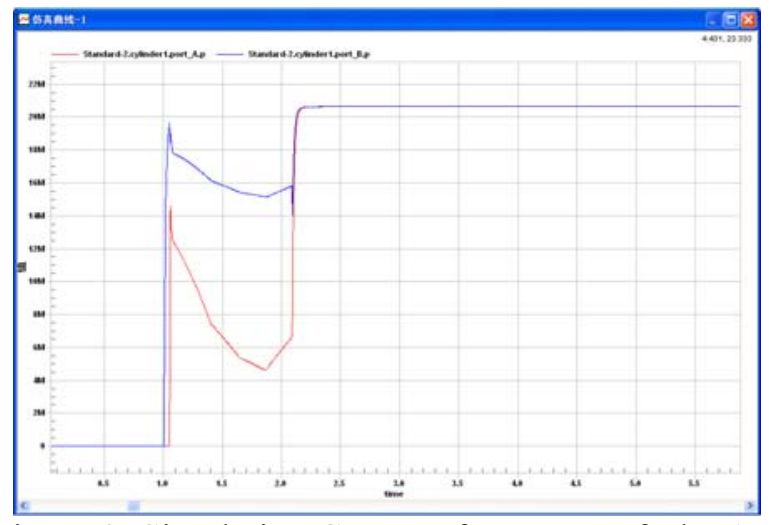

Figure 9: Simulation Curves of Pressure of The Actuator

This HIL simulation system has simple structure and low cost. Through the simulation of ARJ21 aircraft thrust reverser hydraulic system, we can verify the control logic in various working conditions, evaluate key performance of the system, so that the number and cost of the tests can be reduced, and the optimization of the design of ARJ21 aircraft hydraulic system and tests can be provided with basis.

\section{Conclusions}

This article demonstrates a Modelica-based HIL simulation solution exclusively developed for aircraft thrust reverser hydraulic system. The HIL simulation system, running on general computer with Windows operating system, communicates with external hardware through serial port. The cost of this HIL simulation system is very low, and its sampling period can be up to $20 \mathrm{~ms}$, so it's especially useful for those situations where very high real-time capability is not required.

The prototype application of the simulation of ARJ21 thrust reverser shows that this HIL simulation system, which uses Modelica language to model aircraft thrust reverser hydraulic system and connects with PLC control system, can greatly increase the efficiency of tests, and reduce the number and the cost of tests.

The future work is to enhance the real-time capability of the simulation with general Windows computer, as well as to use MWorks to generate target code, which can be used in real-time system.

\section{Acknowledgments}

This work was supported by the National Natural Science Foundation of China (Grant No.60704019 and Grant No.60874064).

Special thanks to Medelon Corporation for authorized use of HyLibLight library.

\section{Acronyms}

ARJ21: Advanced Regional Jet for the 21st Century COMAC: Commercial Aircraft Corp. of China, Ltd. CL: Cowl Lock ICV: Isolation Control Valve DCV: Directional Control Valve PLC: Programmable Logic Controller HIL: Hardware-in-the-Loop

\section{References}

[1] Robert A Jones, Thrust reverser. US4373328, $1983,2$.

[2] Peter Fritzson, Engelson Vadim. Modelica a unified object oriented language for system modeling and simulation[A]. Proceedings of the 12th European Conference on Object oriented Programming[C]. 1998, 67 - 90.

[3] Peter Fritzson, Principles of Object-Oriented Modeling and Simulation with Modelica 2.1. Piscataway, NJ: IEEE Press, 2004.

[4] FAN-LI Zhou, LI-PING Chen, YI-ZHONG Wu, JIAN-WAN Ding, JIAN-JUN Zhao, YUN-QING Zhang, MWorks: a Modern IDE for Modeling and Simulation of Multidomain Physical Systems Based on Modelica, Proceedings of the 5th International Modelica Conference, Volume 2, 725-732, 2006. 